\title{
Long-wave infrared spectral filter with semiconductor materials
}

\author{
Clément Maës ${ }^{\mathrm{a}, \mathrm{b}}$, Grégory Vincent ${ }^{\mathrm{a}}$, Fernando González-Posada Flores ${ }^{\mathrm{b}}$, Laurent Cerutti ${ }^{\mathrm{b}}$, Riad \\ Haïdar $^{\mathrm{a}}$, Thierry Taliercio ${ }^{\mathrm{b}}$ \\ ${ }^{a}$ ONERA The French Aerospace Lab, Chemin de la Hunière, 91123 Palaiseau Cedex, France ; \\ bUniv. Montpellier, CNRS, IES, UMR 5214, F-34000 Montpellier, France
}

\begin{abstract}
We present a theoretical study of a nanostructured guided-mode resonant (GMR) spectral filter operating in the long-wave infrared (LWIR) wavelength range. The component is made of III-V semiconductors: heavily n-doped InAsSb for the grating and $\mathrm{GaSb}$ for the waveguide of the GMR resonator. In order to study the tolerance for the fabrication process and to adjust the resonance of the filter, a geometric study is also presented.
\end{abstract}

Keywords: nanophotonic, plasmonics, spectral filters, infrared, semiconductor, guided-mode resonant, adjustability of the resonnance

\section{INTRODUCTION}

In the context of infrared spectral filtering, nanostructured components offer simple filter architectures such as GMR [1], usually made of metallic or dielectric materials. The current trend is the integration of the filter right on the detection pixel and Bierret et al. demonstrated theoretically in Ref. [2] that the concept of metallo-dielectric GMR filters is good candidates for this integration which could simplify the imaging system.

The integration of metallo-dielectric components directly on an infrared detection pixel is complicated because it requires additional technological processes that may not be compatible with the fabrication process of detectors. Indeed the main causes are the materials involved and to the compatibility between metals and semiconductors

We have shown in Ref. [3] that we can replace the metallic part of the GMR with a silicon heavily doped semiconductor (HDS), n-doped InAsSb:Si. Thus we can realize a monolithic integration using only semiconductors and we have also demonstrated experimentally a GMR spectral filter made of III-V semiconductors.

In this article, we first present a GMR filter made of InAsSb and GaSb, which are the materials used in particular for superlattices photodetectors [4]. Then we show the possibility of modifying the resonance wavelength of the GMR spectral filter with a geometric study which is interresting for multispectral imaging purpose.

\section{GMR ARCHITECTURE}

The architectures of GMR components are composed of a waveguide, place of the resonance, and a grating allowing the coupling between the incident wave and the guided mode thanks to the $+/-1$ orders diffracted by the grating in the thin film (Figure 1.). Such architectures have band-pass or band-cut filtering capabilities, depending on the materials and the geometry.

Due to the diffractive nature of the coupling grating, standard GMR components are sensitive to the angle of incidence of light. However, with carefull design of the grating, angular acceptance can be ignored [5]. For non-zero incidence, two resonances are produced (symmetric and antisymmetric) at different wavelengths.

Usualy, the materials used for these structures are dielectric materials where quality factors are high, and losses are low [6] but a metal can also be used for the grating in order to widen the bandwidth, but it leads to losses [7].

The deposition of this type of component on a detection pixel the technology is complex when we use materials as dielectric or metal because we are obliged to make a detection pixel with epitaxial growth and then to deposit the filter with another deposition process. 


\section{SEMICONDUCTOR GMR}

In a first step, we aim at determining geometrical and optical parameters of the component to achieve spectral filtering. The grating of the GMR filter is made of heavily doped InAsSb, while the waveguide is made of undoped GaSb and we use a GaAs substrate. We define a component geometry operating in the far infrared spectral range ( $8 \mu \mathrm{m}-14 \mu \mathrm{m}, \mathrm{LWIR})$, where the heavily doped InAsSb behaves as a metal [3] (doping level is $\mathrm{N}=5.5 \mathrm{e}^{-19 \mathrm{~cm}^{-3}}$ ).

To do so, we need to know the permittivity of the materials involved and we use a Drude model to describe the permittivity:

$$
\varepsilon(\omega)=\varepsilon_{\infty}\left(1-\frac{\omega_{p}^{2}}{\omega(\omega+i \gamma)}\right)
$$

where $\varepsilon_{\infty}, \gamma$ and $\omega_{p}$ correspond to the dynamic dielectric permittivity, the scattering rate, and the screened plasma frequency, respectively. For HDS, screened plasma frequency is defined as:

$$
\omega_{p}=\sqrt{\frac{N e^{2}}{\varepsilon_{0} \varepsilon_{\infty} m_{e f f}}}
$$

with $m_{e f f}$ being the effective mass and $\mathrm{N}$ being the doping level (we assume that dopant atoms are fully ionized).

Undoped GaSb is transparent in the LWIR and we have taken a GaAs substrate because it has a refractive index of 3.27, lower than that of GaSb, which is essential to guide the wave. For GaSb, we use $\mathrm{n}_{\mathrm{GaSb}}=3.77$ based on experimental work by Roux et al. [10].

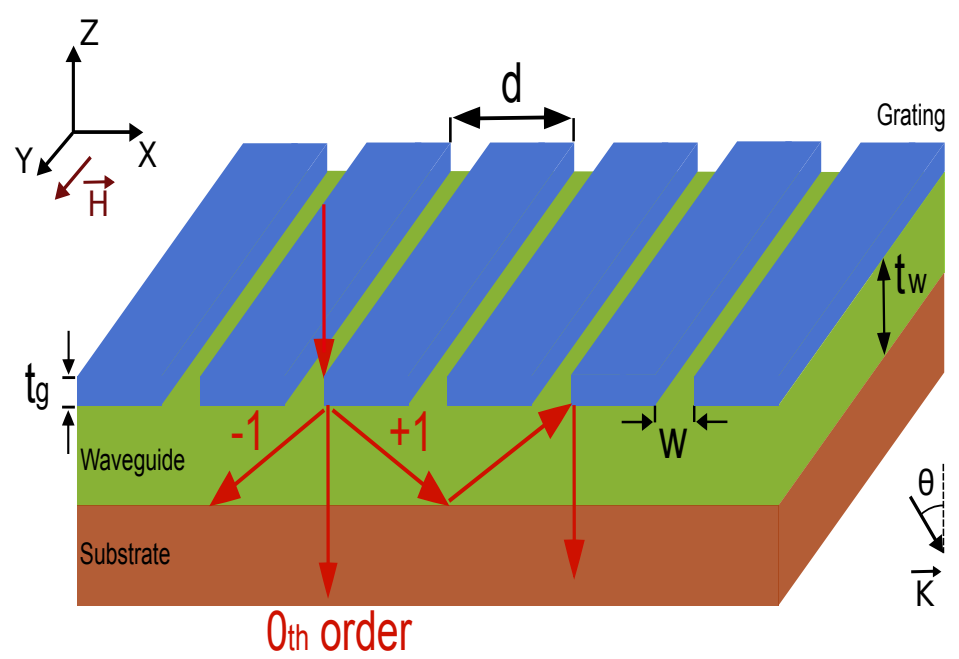

Figure 1. Guided-mode resonance filter.

With Figure 2., we show a normal incidence transmission spectrum calculation, in transverse magnetic polarization. This simulation result is obtained using RCWA (Rigorous coupled-wave analysis) [11]. We observe a resonant transmission at $\lambda=11.88 \mu \mathrm{m}$, with a maximum transmission $\mathrm{T}_{\max }=86.75 \%$ in the GaAs substrate. The permittivity of the InAsSb was modeled with a Drude function, where we took into account an effective mass of $0.127 \mathrm{~m}_{\mathrm{o}}$ because of large carrier density. We have evaluated it, thanks to reflection measurements performed on an unstructured $\mathrm{GaAs}-\mathrm{GaSb}-\mathrm{n}^{++}-$ InAsSb stack [9]. For n-InAsSb, we set $\varepsilon_{\infty}=10.4, \gamma=1 \mathrm{e} 13 \mathrm{rad}^{-1}{ }^{-1}$ [8] and the screened plasma frequency $\omega_{p}$ is experimentally determined by measuring the Brewster mode in function of the doping level [9]: we have $\omega_{p}=3.5847 \mathrm{e} 14$ rad. $\mathrm{s}^{-1}$ for $\mathrm{N}=5.5 \mathrm{e} 19 \mathrm{~cm}^{-3}$. 


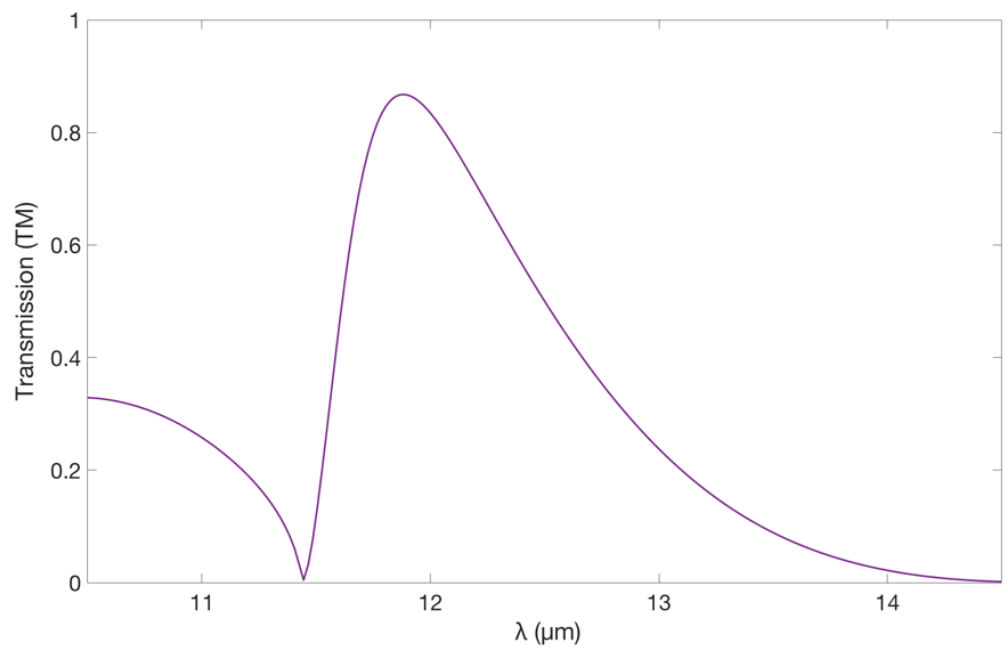

Figure 2. Transmission spectrum calculated in TM polarization and under normal incidence $\left(\mathrm{d}=3.5 \mu \mathrm{m} ; \mathrm{w}=1.2 \mu \mathrm{m} ; \mathrm{t}_{\mathrm{g}}=1.5 \mu \mathrm{m}\right.$ and $\left.\mathrm{t}_{\mathrm{w}}=2.2 \mu \mathrm{m}\right)$.

A similar GMR filter with HDS has already been fabricated and characterized by our team [3]. Complementary studies on the angular dependence and the temperature dependence have also been performed and are presented in Ref. [12].

\section{ADJUSTABLE SEMICONDUCTOR GMR}

The filter presented previously has a resonance wavelength of $\lambda_{\mathrm{r}}=11.88 \mu \mathrm{m}$. In order to study the modulation of the spectral position of the resonance in the long-wave infrared band, we present a study concerning the period and the slits width of the grating. Indeed, whereas thicknesses of the layers are defined by epitaxy, these parameters can be adjusted by lithography in the scope of spectrometry or spectro-imaging [13]. Then we also study the dependence of the resonance wavelength and the maximum of the transmission with the waveguide thickness. This study is interesting for the fabrication process.

To do so, we first consider the same parameters (doping level, thicknesses, permittivity, ...) and we modify the value of the period, $d$, without modifying the slits width, $w$. With Figure 3, we present the results of the transmission spectra calculated in TM polarization and under normal incidence for various periods. The calculations are done with the same RCWA method as for the transmission spectrum presented Figure 2 [10]. 


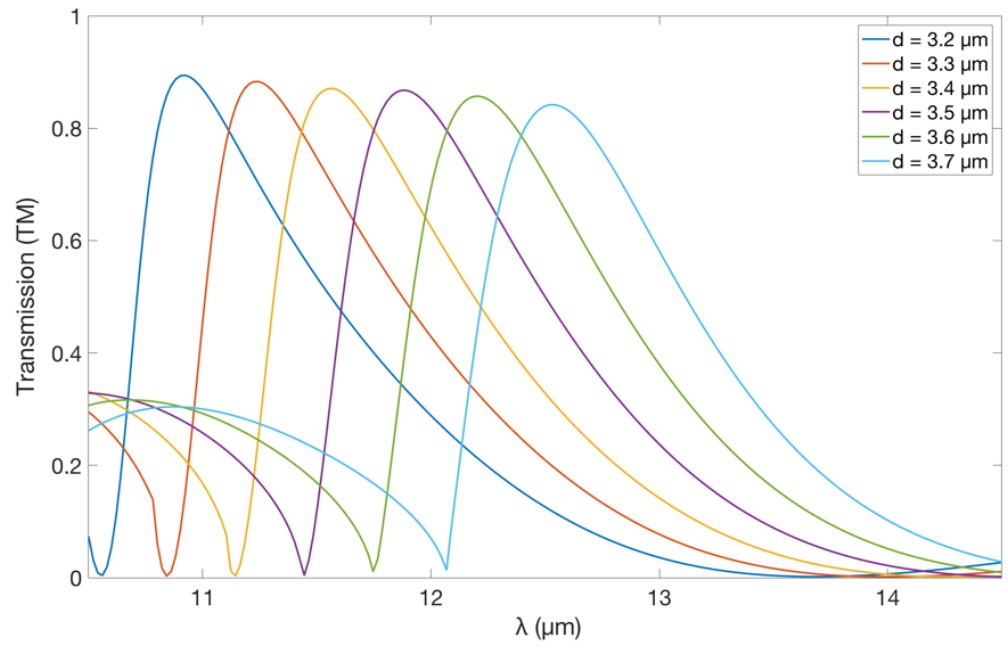

Figure 3. Transmission spectra calculated in TM polarization and under normal incidence for various period $d\left(\mathrm{~N}=5.5 \mathrm{e} 19 \mathrm{~cm}^{-3}\right.$; $\mathrm{w}=1.2 \mu \mathrm{m} ; \mathrm{t}_{\mathrm{g}}=1.5 \mu \mathrm{m}$ and $\left.\mathrm{t}_{\mathrm{w}}=2.2 \mu \mathrm{m}\right)$.

The Table 1. presents the transmission maximum, $T_{\max }$, and the resonance wavelength, $\lambda_{r}$, for each filter designed and defined by the value of the period of the grating, $d$.

Table 1. Properties of the filters under TM polarization for various periods of the gratind $d$.

\begin{tabular}{|l|r|r|r|r|r|r|}
\hline$d(\mu \mathrm{m})$ & 3.2 & 3.3 & 3.4 & 3.5 & 3.6 & 3.7 \\
\hline$T_{\max }(\%)$ & 89.32 & 88.3 & 87.08 & 86.75 & 85.7 & 84.12 \\
\hline$\lambda_{r}(\mu \mathrm{m})$ & 10.9 & 11.24 & 11.56 & 11.88 & 12.2 & 12.55 \\
\hline
\end{tabular}

Figure 3 and Table 1 let us see that when the period of the grating increases, the resonance wavelength is redshifted (for a period $\mathrm{d}=3.2 \mu \mathrm{m}, \lambda_{r}=10.9 \mu \mathrm{m}$ and for $\mathrm{d}=3.7 \mu \mathrm{m}$ we have $\lambda_{r}=12.55 \mu \mathrm{m}$ ) and the maximum of the transmission decreases slightly (from $89.32 \%$ to $84.12 \%$ from $\mathrm{d}=3.2 \mu \mathrm{m}$ to $\mathrm{d}=3.7 \mu \mathrm{m}$ ).

Theses results demonstrate the possibily of adjusting the resonance wavelength with low impact on the transmission maximum and so the capability of the GMR filter. The possibility of modulate the detection in the LWIR is so possible with just modifying a geometric parameter, the period, and we could consider to realize multiple filters.

Then, we do the same study with the slits width of the grating, $w$, without modifying the period, $d$. This study is interesting to predict the tolerance on the slits width during the fabrication process. Indeed the InAsSb layer is thick and the slits width could be so impacted. With Figure 4, we present the results of the transmission spectra calculated in TM polarization and under normal incidence for various slits widths. 


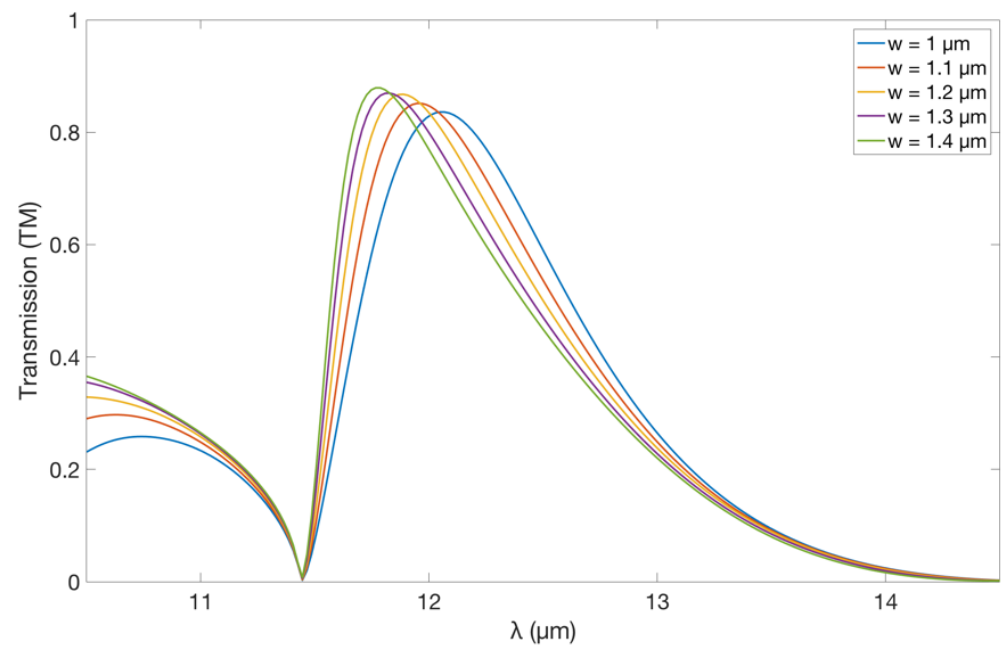

Figure 4. Transmission spectra calculated in TM polarization and under normal incidence for various slits widths $w(\mathrm{~N}=5.5 \mathrm{e} 19$ $\mathrm{cm}^{-3} ; \mathrm{d}=3.5 \mu \mathrm{m} ; \mathrm{t}_{\mathrm{g}}=1.5 \mu \mathrm{m}$ and $\left.\mathrm{t}_{\mathrm{w}}=2.2 \mu \mathrm{m}\right)$.

The Table 2. presents the transmission maximum, $T_{\max }$, and the resonance wavelength, $\lambda_{r}$, for each filter designed and defined by the value of the slits width of the grating, $w$.

Table 2. Properties of the filters under TM polarization for various slits widths of the gratind $w$.

\begin{tabular}{|l|r|r|r|r|r|}
\hline$w(\mu \mathrm{m})$ & 1 & 1.1 & 1.2 & 1.3 & 1.4 \\
\hline$T_{\max }(\%)$ & 83.3 & 84.88 & 86.3 & 86.97 & 87.89 \\
\hline$\lambda_{r}(\mu \mathrm{m})$ & 12.04 & 11.96 & 11.86 & 11.82 & 11.78 \\
\hline
\end{tabular}

Figure 4 and Table 2 let us see that when the slits width of the grating increases, the resonance wavelength is blueshifted (for a slits width $\mathrm{w}=1 \mu \mathrm{m}, \lambda_{r}=12.04 \mu \mathrm{m}$ and for $\mathrm{w}=1.4 \mu \mathrm{m}$ we have $\lambda_{r}=11.78 \mu \mathrm{m}$ ) and the maximum of the transmission increases slightly (from $83.3 \%$ to $87.89 \%$ from $d=1 \mu \mathrm{m}$ to $d=1.4 \mu \mathrm{m}$ ).

By modifying the slits width of the grating, we can also adjust the position of the resonance without impacting the maximum of the transmission. Nevertheless, the effect is less important than with the period of the grating and this parameter is more tolerant concerning the fabrication of the filter.

3.

Last, we have done a study on the waveguide thickness, $t_{w}$, presented with the following Figure 5 and the Table 


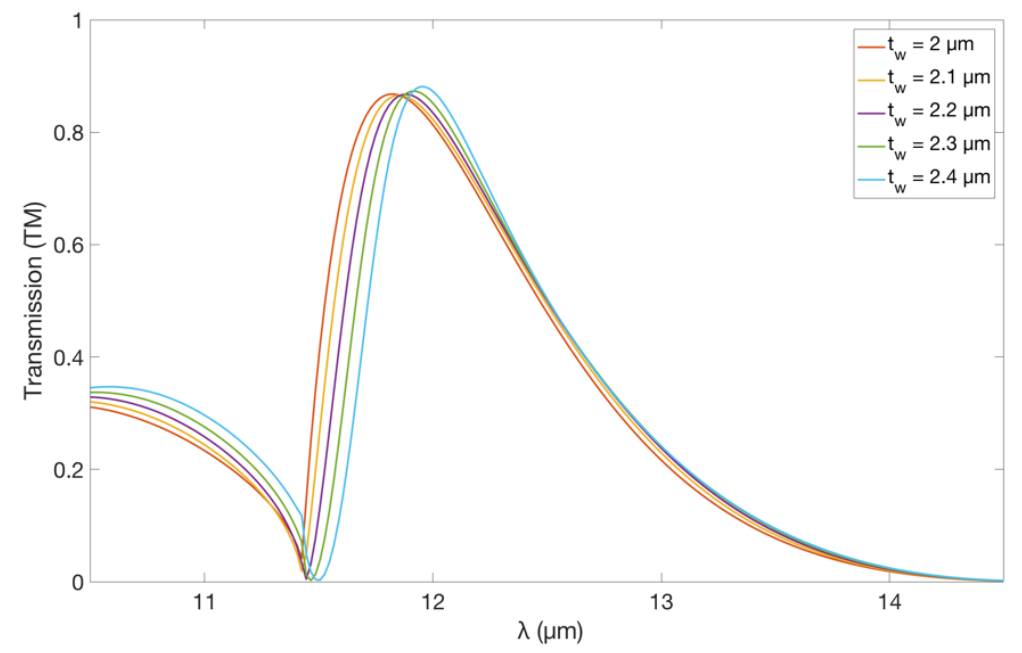

Figure 5. Transmission spectra calculated in TM polarization and under normal incidence for various waveguide thicknesses $t_{w}$ $\left(\mathrm{N}=5.5 \mathrm{e} 19 \mathrm{~cm}^{-3} ; \mathrm{d}=3.5 \mu \mathrm{m}, \mathrm{w}=1.2 \mu \mathrm{m} ; \mathrm{t}_{\mathrm{g}}=1.5 \mu \mathrm{m}\right)$.

Table 3. Properties of the filters under TM polarization for various waveguide thicknesses $t_{w}$.

\begin{tabular}{|l|r|r|r|r|r|}
\hline$t_{w}(\mu \mathrm{m})$ & 2 & 2.1 & 2.2 & 2.3 & 2.4 \\
\hline$T_{\max }(\%)$ & 86.80 & 86.57 & 86.75 & 87.31 & 88.12 \\
\hline$\lambda_{r}(\mu \mathrm{m})$ & 11.82 & 11.84 & 11.86 & 11.9 & 11.94 \\
\hline
\end{tabular}

With this study on the waveguide thickness, we can also observe a small impact on the resonance : for a waveguide thickness $\mathrm{t}_{\mathrm{w}}=2 \mu \mathrm{m}$ we have $\lambda_{r}=11.82 \mu \mathrm{m}$ and $\mathrm{T}_{\max }=86.80$ and for $\mathrm{t}_{\mathrm{w}}=2.4 \mu \mathrm{m}$ we have $\lambda_{r}=11.94 \mu \mathrm{m}$ and $\mathrm{T}_{\max }=88.12$.

This study on the geometrical parameters allows us to observe a good tolerance concerning the slits width of the grating and the waveguide thickness, and the potential that offers the variation the period to adjust the resonance position.

\section{CONCLUSION}

In conclusion, we proposed the design of an all-semiconductor spectral filter based on GMR structure for the longwave infrared. This study demonstrates the possibility to realize surface nanostructured spectral filters with materials fully compatible with epitaxial growth of T2SL photodetectors.

Moreover we have shown a simple approach to realize adjustability in the long-wave infrared band with this GMR filter by modifying the period of the gratting. The study of the slits width of the grating and the waveguide thickness demonstrates a good tolerance for these two parameters and is usefull for the fabrication process.

This work has been partially funded by French Investment for the Future Program (EquipEx EXTRA, ANR 11EQPX-0016).

\section{REFERENCES}

[1] Wang, S. S., Magnusson, R., Bagby, J. S., \& Moharam, M. G. (1990). Guided-mode resonances in planar dielectric-layer diffraction gratings. JOSA A, 7(8), 1470-1474.

[2] Bierret, A., Vincent, G., Jaeck, J., Pelouard, J. L., Haïdar, R., \& Pardo, F. (2017). Field extension inside guidedmode-resonance filters under a focused beam. Optics letters, 42(20), 4187-4190. 
[3] Maës, C., Vincent, G., Flores, F. G. P., Cerutti, L., Haïdar, R., \& Taliercio, T. (2019). Infrared spectral filter based on all-semiconductor guided-mode resonance. Optics letters, 44(12), 3090-3093.

[4] Rodriguez, J. B., Christol, P., Cerutti, L., Chevrier, F., \& Joullié, A. (2005). MBE growth and characterization of type-II InAs/GaSb superlattices for mid-infrared detection. Journal of Crystal Growth, 274(1-2), 6-13.

[5] Sakat, E., Héron, S., Bouchon, P., Vincent, G., Pardo, F., Collin, S., ... \& Haïdar, R. (2013). Metal-dielectric biatomic structure for angular-tolerant spectral filtering. Optics letters, 38(4), 425-427.

[6] Fehrembach, A. L., Lemarchand, F., Talneau, A., \& Sentenac, A. (2010). High Q Polarization Independent Guided-Mode Resonance Filter With "Doubly Periodic" Etched Ta \$_2 \$ O \$ 5 \$ Bidimensional Grating. Journal of Lightwave Technology, 28(14), 2037-2044.

[7] Sakat, E., Vincent, G., Ghenuche, P., Bardou, N., Collin, S., Pardo, F., ... \& Haïdar, R. (2011). Guided mode resonance in subwavelength metallodielectric free-standing grating for bandpass filtering. Optics letters, 36(16), 3054-3056.

[8] Milla, M. J., Barho, F., González-Posada, F., Cerutti, L., Bomers, M., Rodriguez, J. B., ... \& Taliercio, T. (2016). Localized surface plasmon resonance frequency tuning in highly doped $\mathrm{InAsSb} / \mathrm{GaSb}$ one-dimensional nanostructures. Nanotechnology, 27(42), 425201.

[9] Taliercio, T., Guilengui, V. N., Cerutti, L., Tournié, E., \& Greffet, J. J. (2014). Brewster "mode" in highly doped semiconductor layers: an all-optical technique to monitor doping concentration. Optics express, 22(20), 2429424303.

[10] Roux, S., Barritault, P., Lartigue, O., Cerutti, L., Tournié, E., Gérard, B., \& Grisard, A. (2015). Mid-infrared characterization of refractive indices and propagation losses in GaSb/AlXGa1- XAsSb waveguides. Applied Physics Letters, 107(17), 171901.

[11] Hugonin, J.P. and Lalanne, P., "Reticolo software for grating analysis", Institut d'Optique, Orsay, France (2005).

[12] Maës, C., Vincent, G., Flores, F. G. P., Cerutti, L., Haïdar, R., \& Taliercio, T. (2019, September). Semiconductorbased nanostructures for spectral filtering. In Plasmonics: Design, Materials, Fabrication, Characterization, and Applications XVII (Vol. 11082, p. 110821T). International Society for Optics and Photonics.

[13] Haïdar, R., Vincent, G., Collin, S., Bardou, N., Guérineau, N., Deschamps, J., \& Pelouard, J. L. (2010). Freestanding subwavelength metallic gratings for snapshot multispectral imaging. Applied Physics Letters, 96(22), 221104. 